\section{Spontaneous closure of retinal pigment epithelium microrip in the natural course of central serous chorioretinopathy}

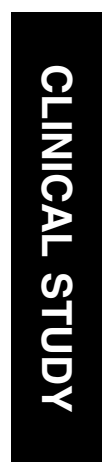

\begin{abstract}
Purpose To study the natural course of changes observed in the retinal pigment epithelium (RPE) on spectral domain OCT transverse and RPE fit C-scans corresponding to the leakage point observed on fundus fluorescein angiograms in central serous chorioretinopathy (CSC).

Methods Thirteen patients with acute CSC were prospectively enrolled and followed up for 6 months. All were examined using Zeiss Cirrus HD-OCT Model 4000. Transverse and RPE fit C-scans corresponding to point of leakage were superimposed on an OCT fundus photograph and correlated with the leakage point on a fluorescein angiogram. The RPE alterations observed at the leakage point were noted at presentation and follow-up.
\end{abstract}

Results Transverse and RPE fit C-scans showed an irregularity of RPE resembling a 'honeycomb' pattern in $11(85 \%)$ eyes at the point corresponding to the fluorescein leakage site. These were observed as pigment epithelium detachment (PED) on Raster line scan. Six of these 11 eyes $(54.5 \%)$ showed a disruption/breach in the RPE on transverse C-scan and on RPE fit C-scan. All eyes showed a resolution of subretinal fluid with a closure of microrip, and the honeycombed pattern at the leakage site was replaced with RPE hypertrophy.

Conclusion RPE microrips show spontaneous closure in the natural course of CSC.

Eye (2010) 24, 595-599; doi:10.1038/eye.2009.193; published online 31 July 2009

Keywords: central serous chorioretinopathy; pigment epithelium detachment; spectral domain optical coherence tomography

V Gupta, P Gupta, MR Dogra and A Gupta
Central serous chorioretinopathy (CSC) is a disease of unknown etiology, which is postulated to result from abnormalities in choroidal circulation, which, in turn, makes the overlying retinal pigment epithelium (RPE) dysfunctional, resulting in the development of serous retinal detachment. ${ }^{1-3}$ The increased hydrostatic pressure caused by hyperpermeable choroid is believed to push the RPE forward, resulting in the development of pigment epithelium detachment (PED). Further, a microrip or 'blow-out' in this PED has been postulated to result in a focal leak, which is observed as the point of leak on fluorescein angiogram. ${ }^{4-6}$ Recently, a RPE defect within the PED at the leakage site has been reported on both time-domain ${ }^{7}$ and fourier-domain OCT. ${ }^{8}$ Fujimoto et $a l^{8}$ observed RPE abnormalities at the site of leakage in $96 \%$ of eyes, with $22 \%$ of the eyes showing an RPE defect that was successfully treated with thermal laser photocoagulation. However, the natural course of these RPE abnormalities observed in the acute phase of CSC is not known.

The new Cirrus HD-OCT Model 4000, using advanced imaging technology (spectral domain), acquires data 70 times faster than the time-domain conventional OCT, with a transverse and axial resolution of $15 \mu \mathrm{m}$ and $5 \mu \mathrm{m}$, respectively. The multi-planar reformat (MPR) enables data acquisition in whole cubes and also gives en face images or C-scans that show a horizontal slice of retina with orientation parallel to the surface of the retina. Linescanning laser ophthalmoscope (LSLO) simultaneously captures the OCT fundus, giving a clear image of the retinal area addressed by the scan. The image display option makes it possible to overlay C-scan/RPE fit scan on the OCT fundus for exact localization
Advanced Eye Centre, Post Graduate Institute of Medical Education and Research, Chandigarh, (UT)، India

Correspondence: V Gupta, Advanced Eye Centre, Department of Ophthalmology, Post Graduate Institute of Research, Chandigarh, (UT) 160012, India

Tel: 91172274 7837;

Fax: 91-172-2747837.

E-mail: eyepgi@

sify.com

Received: 3 April 2009 Accepted in revised form: 8 June 2009 Published online: 31 July 2009 Medical Education and 
of the pathology. These features make it an ideal tool for studying the structural alterations in the RPE corresponding to any specific area of interest in the retina. OCT images, however, are generated by reflections from regions in which refractive index changes for the wavelengths used and different layers are identified using segmentation software. The images obtained are therefore correlates of the underlying anatomy and not direct images. In this study, we used this technology to study morphological alterations in RPE corresponding to the leakage point observed on fundus fluorescein angiograms in a series of patients with acute CSC at presentation. We documented the natural course of these alterations in RPE in these patients, all of whom showed a spontaneous resolution of CSC during follow-up.

\section{Materials and methods}

All patients with serous retinal detachment due to CSC in at least one eye, observed between October 2007 and January 2008, had their OCT scans carried out on Cirrus HD-OCT Model 4000 (Carl Zeiss Meditec, Dublin, CA, USA). All patients had a fundus fluorescein angiography (Visupac, 450 Plus, Carl Zeiss, Jena, Germany) performed on the same day. Acquisition protocols for Cirrus HD-OCT included 5-line Raster scans, Macular cube 200X 200 and Macular cube 512 X128 combo. The 5-line Raster scan consists of five horizontal lines of $6 \mathrm{~mm}$ length, separated $0.25 \mathrm{~mm}$ from each other (default), so that together they cover a $1 \mathrm{~mm}$ width. This scan gives the greatest resolution. The Macular cube 512 X128 combo generates a cube of data through a $6 \mathrm{~mm}$ square grid by acquiring a series of 128 horizontal line scans, each composed of 512 A-scans, whereas the Macular cube 200 X200 combo generates a cube of data through a $6 \mathrm{~mm}$ square grid by acquiring a series of 200 horizontal line scans, each composed of 200 A-scans. Patients with a history of thermal laser photocoagulation or any intravitreal injection were excluded.

High definition image analysis (HDIA) was carried out by viewing line scans of the 5-line Raster scan. The leakage point in the dye transit phase in patients with serous detachment was noted on fluorescein angiogram and the corresponding point was identified on the OCT fundus image for placement of the slice navigator. The OCT fundus image is observed on the upper left viewport of the screen during analysis and is captured simultaneously by LSLO, thus giving a clear image of the retinal area being addressed by the scan. Slice navigators are horizontal and vertical lines that can be placed on the area of interest on the OCT fundus image so as to obtain the OCT scan of the desired point. To ensure a proper registration of images, the point of interest was marked on the OCT fundus photograph and matched with fluorescein angiogram using retinal vessels as landmarks. Slice navigators were positioned on the area of interest, and the corresponding point on Raster line scans, single-layer RPE map and 3-D volume-rendered image was studied.

An advanced interactive analysis for each eye was carried out by creating a slab or slice through the cube top with the development of en face or C-scans. The en face or C-scans include a horizontal slice of retina with orientation parallel to the surface of the retina and gives the same perspective as does the fundus image. Slice navigator was used to select the area of leak on the OCT fundus photograph or PED on the line scan. The corresponding changes in RPE were studied on the en face scan (slice that gives the flat plane), which was then superimposed on the LSO fundus image to match it with the point of leak. After obtaining transverse C-scans, RPE fit was applied to adapt C-scan to the specific curve of the patient's eye, so that the resultant $\mathrm{C}$-scans were confined to the RPE plane. On the basis of the retina's curvature, the Cirrus OCT can calculate and present a layer called RPE fit, which represents a normal parabolic RPE for an eye. Geometry dictates that RPE may form a ring because of ocular curvature, as the tissue may falsely bend up or down. To overcome this, we applied an 'RPE fit' option that fits a line to the general curvature of RPE, thus adapting the C-scan to the specific curve of the patient's eye, with the resultant C-scan being confined to a distinct anatomic plane. The RPE fit line can be used to view variations from normal in the actual RPE contour. The level and thickness of the RPE fit was adjusted as per the height of leakage point. In addition, 3-D volume rendering was carried out that allowed the full-screen display of entire data cubes in 3-D, and selection of RPE checkbox showed the selected RPE surface as a single-colour contour. Two scans were performed on each eye and the one with the best signal strength was considered for the final analysis. The patients were followed up for 6 months or till the resolution of the subretinal fluid (whichever was earlier). Three experts (VG, PG, and AG), with experience in interpreting OCT images, independently evaluated the OCT scans and compared them with the fluorescein angiogram. All the scans were viewed and also evaluated thrice by each expert to assess the consistency of analysis, including analysis on different days. All the experts agreed on all scans. We certify that all applicable institutional and governmental regulations with regard to the ethical use of human volunteers were followed during this research. 


\section{Results}

The study included eight men and five women with age ranging between 35 and 61 years. Only one eye with acute CSC was studied. None of the eyes had a previous CSC. Fundus fluorescein angiogram (FFA) showed an expanding dot in $10(76 \%)$ and a smokestack sign in 3 (24\%) eyes. Raster line scans showed neurosensory detachment, observed as a hyporeflective area between RPE and sensory retina in all 13 eyes. In addition, Raster line scans showed well-defined PED in 11(77\%) eyes. Morphological alterations of RPE at the leakage point included an irregularity (undulating pattern) in the area of PED in $8(62 \%)$ eyes on Raster line scans, and a breach in RPE in $3(23 \%)$ eyes. Study of the transverse C-scans, including RPE fit, which were obtained corresponding to the areas of pathology, revealed serous retinal detachment as an area of hyporeflectivity between the RPE and neurosensory retina in all the 13 eyes and PED in $11(77 \%)$ eyes. Morphological alterations in RPE corresponding to the leakage point, including the irregularity or RPE bump projecting into the overlying serous detachment, were observed in 11 eyes (85\%), both on transverse C-scans and RPE fit. Overall, six eyes (46\%) showed a microrip within the area of irregular RPE on transverse C-scan. The corresponding point had a 'honeycombed' appearance on RPE fit scans. On comparing the OCT fundus image C-scan /RPE fit overlays with the corresponding fluorescein angiogram, the location of the RPE breach/ 'honeycombed' pattern was found to correspond to the leakage point in all eyes (Figures 1,2). The RPE bump also corresponded to the leak on the fluorescein angiogram.

On follow-up, the subretinal fluid resolved in all the eyes, the PED that was observed in 11 eyes at presentation resolved in five eyes $(45.5 \%)$, decreased in size in three eyes $(27.2 \%)$, and persisted in three eyes (27.2\%). Resolution of PED was accompanied by hypertrophy of RPE with a microrip closure at the site. The honeycombed pattern at the leakage site was replaced with hypertrophy of RPE in all the seven eyes on follow-up (Figures 3-6).

\section{Discussion}

The pathophysiology of CSC still remains unclear. FFA shows a focal RPE leak with pooling of the dye in the overlying serous retinal detachment. ${ }^{4}$ The morphological alterations occurring at the site of focal leak have been studied and reported to show RPE abnormalities including an RPE bulge in the posterior pole, ${ }^{9} \mathrm{RPE}$ detachments, ${ }^{9-13}$ reproducible small RPE bulge (which researchers did not consider to be PEDs or artifactual), ${ }^{11}$ irregular lesions of $\mathrm{RPE},{ }^{14}$ and more recently, RPE disruption indicating an anatomical microrip of RPE. ${ }^{7}$

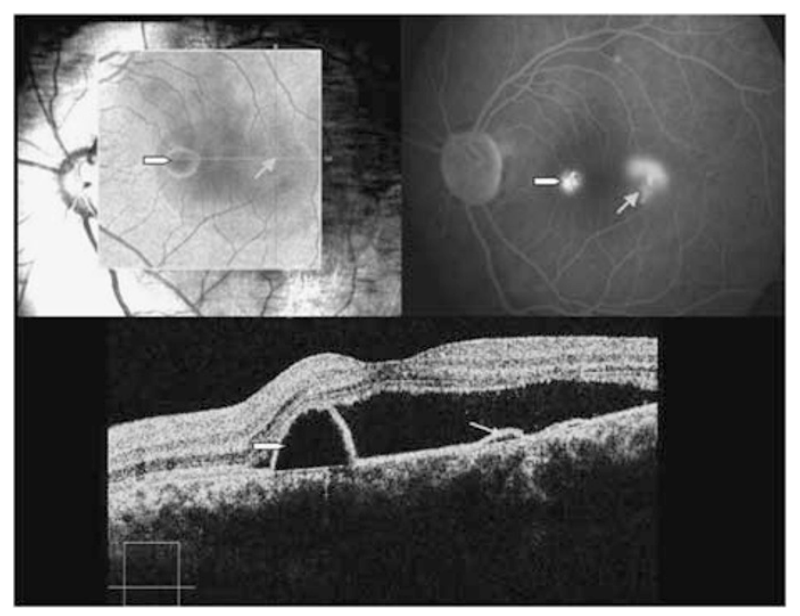

Figure 1 (Patient \#1). OCT fundus photograph of the left eye showing an area of pigment epithelium detachment (PED) (thick arrow) and a possible leakage site (thin arrow) (top left) in a patient with central serous chorioretinopathy. The corresponding fluorescein angiogram shows hyperfluorescence corresponding to PED (thick arrow) and smokestack pattern of leak (thin arrow) (top right). Raster line scan shows dome-like elevation of PED (thick arrow) and irregular undulations of retinal pigment epithelium (RPE) at the leakage site (thin arrow). The RPE breach is not seen in this scan (bottom).

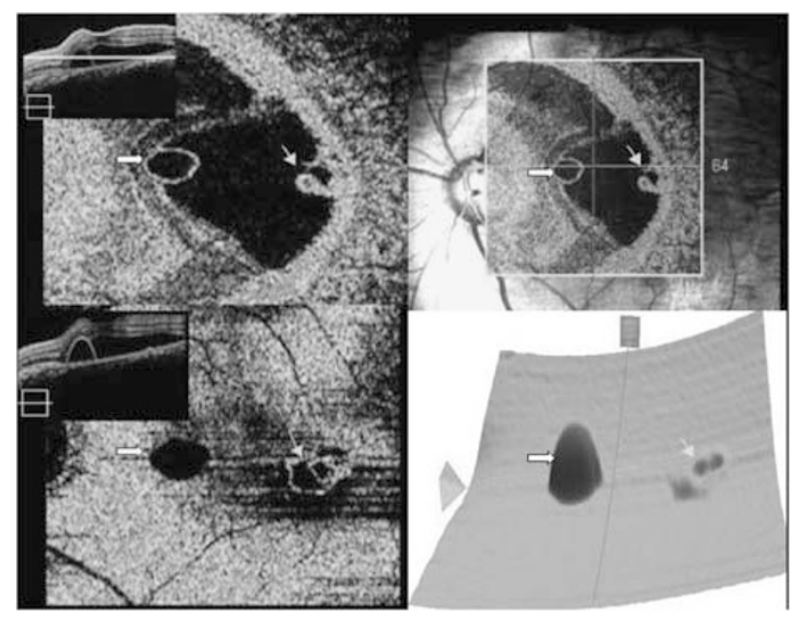

Figure 2 (Patient \#1). Transverse C-scan on passing through the pigment epithelium detachment (PED) and leakage site (inset) shows PED as a complete ring (thick arrow) and retinal pigment epithelium breech corresponding to the point of leak (thin arrow) (top left). Superimposition of C-scan on OCT fundus indicates a correspondence of both PED (thick arrow) and leakage point (thin arrow) (top right). Retinal pigment epithelium (RPE) fit scan C-scan ( $16 \mu$ thick) passing through the bottom of the PED and an irregular retinal pigment epithelium at the leakage site (inset) show a uniform ring corresponding to PED (thick arrow) and a honeycombed appearance at the site corresponding to the leakage point (thin arrow) (bottom left). Single-layer 3-D RPE surface map too shows non-leaking PED as a dome-shaped elevation (thick arrow) and an irregular honeycombed appearance corresponding to the leakage site (thin arrow) (bottom left). 


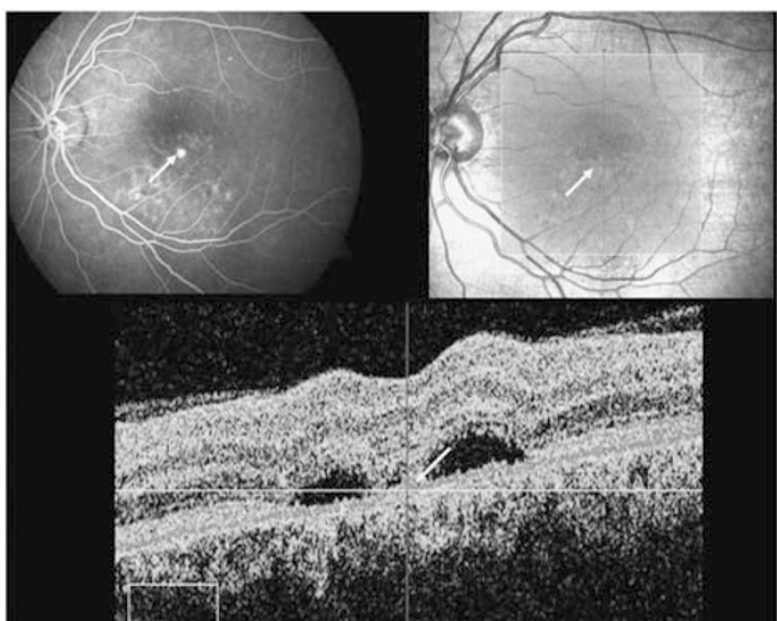

Figure 3 (Patient \#2) Fundus fluorescein angiogram of the left eye of a patient with acute central serous chorioretinopathy (CSC). The arrow indicates the leakage site to be studied (top left). OCT fundus photograph shows the placement of the slice navigator at the point of interest to be studied further (top right) The Raster line scan through the leakage site shows irregular retinal pigment epithelium (RPE) with a microrip (arrow) (bottom left).

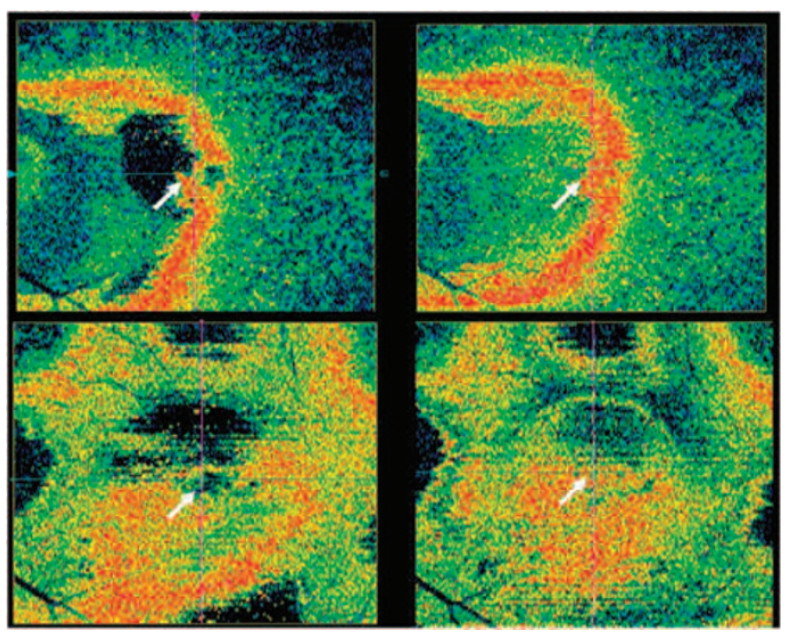

Figure 4 (Patient \#2). Transverse C-scan at presentation, passing through the leakage site, shows the honeycombed appearance with subretinal fluid observed as a hyporeflective area anterior to it (arrow) (top left). Repeat scan through the same area 10 weeks later shows a resolution of the subretinal fluid with a resolution of the honeycombed appearance of retinal pigment epithelium (RPE) (arrow) (top right). RPE fit scan C-scan at presentation shows irregular, honeycombed RPE, with the dark area indicating disruption (arrow) (bottom left). Repeat scan carried out 10 weeks later shows the restoration of the integrity of the RPE layer on follow-up (arrow) (bottom right).

In this series, we used high-definition Spectral domain OCT with an axial resolution of $5 \mu \mathrm{m}$ in a series of patients with CSC to study the morphological alterations

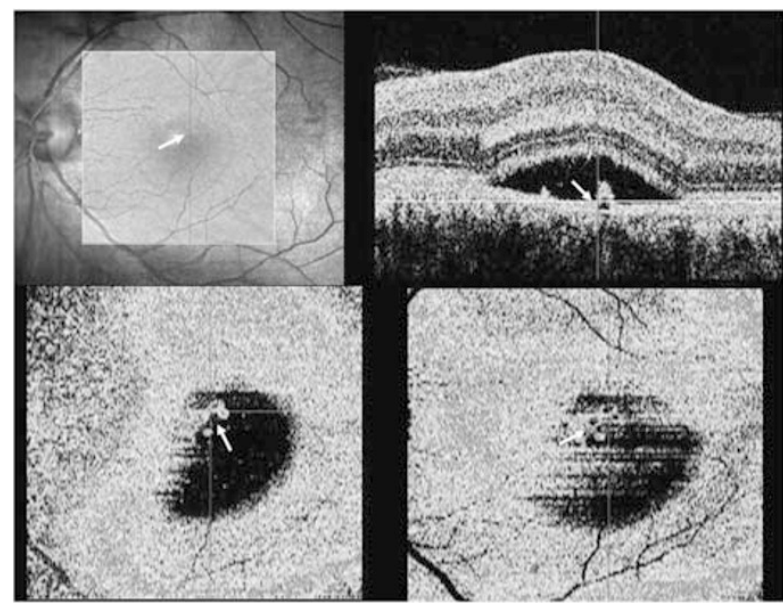

Figure 5 (Patient \#3). OCT Fundus photograph shows the placement of the slice navigator at the point of interest to be studied further (arrow) (top left). Raster line scan through the leakage site shows pigment epithelium detachment (PED) with microrip (arrow) (top right). Transverse C-scan through the same area shows microrip (arrow) (bottom left), whereas retinal pigment epithelium (RPE) fit C-scan shows the honeycombed appearance of RPE (bottom right).

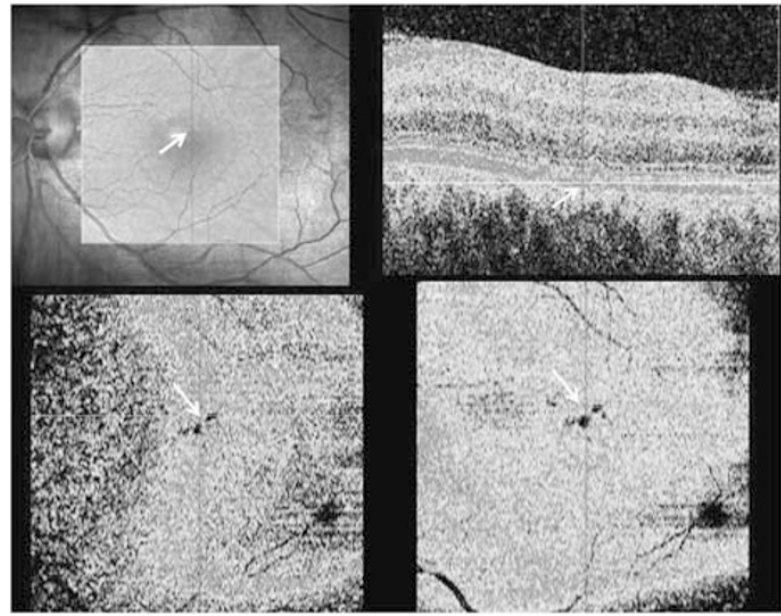

Figure 6 (Patient \#3). OCT Fundus photograph taken 6 weeks later shows the placement of slice navigator at the same point as in Figure 5 (top left). Raster line scan through the leakage site shows small residual pigment epithelium detachment (PED) with closure of microrip and resolution of subretinal fluid (top right). Transverse C-scan (bottom left) and retinal pigment epithelium (RPE) fit C-scans (bottom right) through the same area show hyporeflective ring corresponding to the residual PED with a resolution of the honeycombed pattern.

occurring at the level of RPE at the site of leakage in CSC. We found that $92 \%$ of the eyes showed an irregularity of RPE, with $54 \%$ of the eyes with an irregular RPE demonstrating a breach in RPE on transverse C-scans. The microrip or breach within the irregular RPE, which 
was appreciable on transverse C-scan, had a honeycomb appearance on RPE fit C-scans.

Choroidal capillary and venous hyperpermeability are known to occur in patients with CSC. ${ }^{1-3}$ This may increase choroidal hydrostatic pressures, pushing the overlying RPE forwards and may explain the PED so commonly observed with conventional OCT. ${ }^{10-13}$ Furthermore, it has been shown in an experimental model that focal RPE disruption, in addition to an increased choroidal fluid pressure, is required to trigger the development of serous detachment. ${ }^{15}$ It has been postulated in the past that mechanical stress in CSC may cause RPE to give way at some point, which is observed as a point of leak on fluorescein angiography. ${ }^{4,6}$ The breach will allow the passage of fluid, including particulate matter, into the subretinal space from the underlying hyperemic choroid. This would also mean that the resolution of subretinal fluid could result from decreased effusion from choroid, accompanied by a healing of the RPE microrip. Recently, Fujimoto et al ${ }^{8}$ reported persistent PED at the leakage site in $22 \%$ of cases. The majority of the patients in their study received thermal laser photocoagulation and do not indicate the natural disease course.

However, what remains unanswered is why the character of PED changes at the site of leakage. To begin with we speculate that increased choroidal pressure causes PED, the transverse section of which would show a uniform ring. Further mechanical stress may cause a breach or microrip in the PED, which, in the cross-section would be observed as an RPE defect or honeycomb pattern. This would result in the development of a serous detachment in CSC. Owing to the breach/microrip, fluid pressure equalizes on either side of the RPE, causing a collapse of the PED, which loses its smooth dome-like contour and is now observed as undulations of the RPE on line scan and as an irregularity or honeycomb appearance on transverse C-scan. The CSC would persist, as long as this RPE disruption persists. Once this PED collapses, the collapsing walls might result in the closure of the RPE microrips that would be observed as an RPE irregularity.

The morphological alterations and the RPE breach observed in our study provide further evidence that mechanical disruption at the level of RPE is the most plausible factor responsible for generating the focal leakage point in CSC, and its spontaneous closure may be one of the factors responsible for the spontaneous resolution of CSC.

\section{Conflict of interest}

The authors declare no conflict of interest.

\section{References}

1 Guyer DR, Yannuzzi LA, Slakter JS, Sorenson JA, Ho A, Orlock D. Digital indocyanine green video angiography of central serous chorioretinopathy. Arch Ophthalmol 1994; 112: 1057-1062.

2 Piccolino FC, Borgia L, Zinicola E, Zingirian M. Indocyanine green angiographic findings in central serous chorioretinopathy. Eye 1995; 9: 324-332.

3 Prunte C, Flammer AJ. Choroidal capillary and venous congestion in central serous chorioretinopathy. Am J Ophthalmol 1996; 121: 26-34.

4 Gass JDM. Pathogenesis of disciform detachment of the neuroepithelium II. Idiopathic central serous chorioretinopathy. Am J Ophthalmol 1967; 63: 587-615.

5 Spaide RF, Campeas L, Haas A, Yannuzzi LA, Fisher YL, Guyer DR et al. Central serous chorioretinopathy in younger and older adults. Ophthalmology 1996; 103: 2070-2079.

6 Goldstein BG, Pavan PR. Blow-outs in the retinal pigment epithelium. Br J Ophthalmol 1987; 71: 676-681.

7 Ober MD, Eandi CM, Jampol LM, Fine HF, Yannuzi LA. Focal retinal pigment epithelium breaks in central serous chorioretinopathy. Retin Cases Brief Rep 2007; 1: 271-273.

8 Fujimoto H, Gomi F, Wakabayashi T, Sawa M, Tsujikawa M, Tano Y. Morphologic changes in acute central serous chorioretinopathy evaluated by fourier-domain optical coherence tomography. Ophthalmology 2008; 115(9): 1494-1500.

9 Hirami Y, Tsujikawa A, Sasahara M, Gotoh N, Tamura H, Otani A et al. Alterations of retinal pigment epithelium in central serous chorioretinopathy. Clin Experiment Ophthalmol 2007; 35: 225-230.

10 Iida T, Hagimura N, Sato T, Kishi S. Evaluation of central serous chorioretinopathy with optical coherence tomography. Am J Ophthalmol 2000; 129: 16-20.

11 Montero JA, Ruiz-Moreno JM. Optical coherence tomography characterization of idiopathic central serous chorioretinopathy. Br J Ophthalmol 2005; 89: 562-564.

12 Gupta V, Gupta A, Dogra MR. Atlas: optical coherence tomography of macular diseases and glaucoma. 2nd ed Jaypee Brs Med Publishers (P) Ltd: New Delhi, India, 2006; 72-103.

13 van Velthoven MEJ, Verbraak FD, Garcia PM, Schlingemann RO, Rosen RB, de Smet MD. Evaluation of central serous retinopathy with en face optical coherence tomography. Br J Ophthalmol 2005; 89: 1483-1488.

14 Mitarai K, Gomi F, Tano Y. Three-dimensional optical coherence tomographic findings in central serous chorioretinopathy. Graefe's Arch Clin Exp Ophthalmol 2006; 244: 1415-1420.

15 Marmor MF, Yao X-Y. Conditions necessary for the formation of serous detachment. Experimental evidence from the cat. Arch Ophthalmol 1994; 112: 830-838. 